\title{
Spatiotemporal Dispersion and Wave Envelopes with Relativistic and Pseudorelativistic Characteristics
}

\author{
J. M. Christian, G. S. McDonald, and T.F. Hodgkinson \\ Joule Physics Laboratory, School of Computing, Science and Engineering, Materials \& Physics Research Centre, \\ University of Salford, Salford M5 4WT, United Kingdom \\ P. Chamorro-Posada \\ Departamento de Teoría de la Señal y Comunicaciones e Ingeniería Telemática, Universidad de Valladolid, \\ ETSI Telecomunicación, Campus Miguel Delibes Paseo Belén 15, E-47011 Valladolid, Spain
}

(Received 7 July 2011; published 17 January 2012)

\begin{abstract}
A generic nonparaxial model for pulse envelopes is presented. Classic Schrödinger-type descriptions of wave propagation have their origins in slowly-varying envelopes combined with a Galilean boost to the local time frame. By abandoning these two simplifications, a picture of pulse evolution emerges in which frame-of-reference considerations and space-time transformations take center stage. A wide range of effects, analogous to those in special relativity, then follows for both linear and nonlinear systems. Explicit demonstration is presented through exact bright and dark soliton pulse solutions.
\end{abstract}

DOI: 10.1103/PhysRevLett.108.034101

PACS numbers: 05.45.Yv, 03.30.+p, 42.25.-p, 42.65.Tg

The slowly-varying envelope approximation (SVEA) is universal in the treatment of wave phenomena [1]. It is routinely deployed across a diverse range of contexts, from photonics and quantum mechanics, to hydrodynamics and plasma physics. One is often concerned with describing the envelope modulating a physical wavelike quantity (such as electric field, fluid velocity, or ion density) with an underlying rapidly oscillating carrier component. The SVEA (which assumes that the longitudinal modulation is slow compared to the scale-length of the carrier oscillation) tends to go hand-in-hand with a Galilean boost to a reference frame moving at some characteristic speed. Together, the SVEA and subsequent Galilean boost constitute a mathematical device that has been applied with great success over many decades. An obvious question to pose is: what happens if this device is omitted?

In this Letter, we provide some surprising answers to that seemingly straightforward question. For clarity, a simple nonlinear system, applicable in a wide range of physical contexts, is chosen for analysis. We find that merely accommodating second-order temporal dispersion without the SVEA introduces unexpected features; a study of the generic interplay between spatiotemporal dispersion and nonlinearity uncovers aspects expected to have general implications for pulse characteristics in linear and nonlinear systems. Such a nonparaxial model is derived for a particular context of optical pulses in nonlinear waveguides. Analysis includes Helmholtz soliton theory in the time-domain, where the governing equation differs structurally from those describing Helmholtz soliton beams [2].

Results furnish a mathematical framework beyond that of conventional models and which is rich in spatiotemporal considerations. When spatial and temporal dispersive effects are both at second order, the structure of the model allows many of its predictions to be interpreted within a framework that is closely akin to Einstein's special theory of relativity (pulse propagation problems are rooted in notions of observers and frames of reference). At the heart of our analysis lies a set of space-time operations related to the Lorentz coordinate transformation [3]. Derivation of the optical model involves some well-known steps (e.g., introduction of wave envelopes and Fourier decomposition of the temporal dispersion operator). However, the "SVEA + Galilean boost" device is abandoned and we instead remain in the laboratory frame. Such a choice is clearly allowed physically; it is, after all, the frame in which experiments are typically performed and measurements made [4]. This is a fundamental feature that distinguishes our analysis from many standard treatments [5-8].

For few-picosecond pulses at communication wavelengths in single-mode silica fibres, it is reasonable to assume the SVEA [9]. The same cannot necessarily be said for light in some semiconductors (e.g., $\mathrm{ZnCdSe} / \mathrm{ZnSe}$ superlattices) with spatial material dispersion (an effect allied to dynamics of polaritons in the medium). The seminal analysis of Biancalana and Creatore [10] probed the role of spatial material dispersion in scalar nonlinear pulse physics by deriving a single leading-order contribution term that can describe its effects in certain parameter regimes. This term, proportional to a second-order longitudinal derivative, can combine with the corresponding generic Laplacian term in wave descriptions (that the SVEA discards). A Galilean boost applied to the resulting Helmholtz-type model can be followed by neglecting a cross-derivative term [10] to render analysis similar to that of nonlinear beams [2]. Here, we examine a more general and exact governing equation that captures 
second-order spatiotemporal dispersion without either approximation.

A scalar electric field travelling down the longitudinal axis $z$ of a waveguide can be represented by $E(t, z)=$ $A(t, z) \exp \left[i\left(k_{0} z-\omega_{0} t\right)\right]+$ c.c., where $A(t, z)$ is the envelope, $t$ is time, and c.c. denotes complex conjugate. The optical carrier frequency and wave number are $\omega_{0}$ and $k_{0}=n_{0} \omega_{0} / c$, respectively, where $n_{0}$ is linear refractive index at frequency $\omega_{0}$, and $c$ is the vacuum speed of light. The Fourier transform of the pulse envelope satisfies $\left[\partial^{2} / \partial z^{2}+i 2 k_{0} \partial / \partial z+k^{2}(\omega)-k_{0}^{2}\right] \tilde{A}\left(\omega-\omega_{0}, z\right)=0$, where the propagation constant $k(\omega)$ depends implicitly on the temporal dispersive properties of the waveguide $[9,11]$. Anticipating $\tilde{A}$ to remain localized near $\omega_{0}$, one has $k^{2}\left(\omega-\omega_{0}\right)-k_{0}^{2} \simeq 2 k_{0}\left[k\left(\omega-\omega_{0}\right)-k_{0}\right]$ and $k\left(\omega-\omega_{0}\right)-$ $k_{0} \simeq k_{1}\left(\omega-\omega_{0}\right)+\left(k_{2} / 2\right)\left(\omega-\omega_{0}\right)^{2}, \quad$ where $\quad k_{j} \equiv\left(\partial^{j} k /\right.$ $\left.\partial \omega^{j}\right)_{\omega=\omega_{0}}$ for $j=0-2$. For a Kerr-type nonlinearity, the dimensionless envelope $u$ satisfies

$$
\kappa \frac{\partial^{2} u}{\partial \zeta^{2}}+i\left(\frac{\partial u}{\partial \zeta}+\alpha \frac{\partial u}{\partial \tau}\right)+\frac{s}{2} \frac{\partial^{2} u}{\partial \tau^{2}}+|u|^{2} u=0 .
$$

Normalized space and time coordinates are $\zeta=z / L$ and $\tau=t / t_{p}$, respectively, where $t_{p}$ is a reference pulse duration and $L=t_{p}^{2} /\left|k_{2}\right|$. The sign of group velocity dispersion (GVD) is flagged by $s=-\operatorname{sgn}\left(k_{2}\right)= \pm 1(+1$ for anomalous; -1 for normal), and $\alpha \equiv k_{1} t_{p} /\left|k_{2}\right|$. The spatial dispersion coefficient $\kappa=\kappa_{0}+D$ has two contributions: $\kappa_{0} \equiv 1 / 2 k_{0} L$ is an inherent wave feature, while $D$ is a medium component that can be negative [10]. Finally, $u=A / A_{0}$ where $A_{0} \equiv\left(n_{0} / n_{2} k_{0} L\right)^{1 / 2}$ and $n_{2}$ is the Kerr coefficient.

In conventional pulse physics [1,4-9,11], one assumes an envelope slowly varying in $\zeta$ then neglects the first term in Eq. (1). The linear wave operator may be simplified further by introducing a set of local time coordinates, $\tau_{\text {loc }} \equiv \tau-\alpha \zeta$ and $\zeta_{\text {loc }} \equiv \zeta$. The governing equation then reduces to the canonical nonlinear Schrödinger (NLS) class, namely $\left[i \partial / \partial \zeta_{\text {loc }}+(s / 2) \partial^{2} / \partial \tau_{\text {loc }}^{2}+|u|^{2}\right]$ $u\left(\tau_{\text {loc }}, \zeta_{\text {loc }}\right)=0$, whose solutions describe waves in a frame moving at speed $1 / \alpha$ along the $\zeta$ axis. Conventionally, the term $i \alpha \partial u / \partial \tau$ is thus transformed away by the Galilean boost. In contrast, our analysis shows that the interplay of this contribution with other terms in Eq. (1) can be of fundamental significance.

When implementing the Galilean boost without making the SVEA, one obtains

$$
\begin{gathered}
\kappa \frac{\partial^{2} u}{\partial \zeta_{\mathrm{loc}}^{2}}+i \frac{\partial u}{\partial \zeta_{\mathrm{loc}}}+\frac{1}{2}\left(s+2 \kappa \alpha^{2}\right) \frac{\partial^{2} u}{\partial \tau_{\mathrm{loc}}^{2}} \\
-2 \kappa \alpha \frac{\partial^{2} u}{\partial \zeta_{\mathrm{loc}} \partial \tau_{\mathrm{loc}}}+|u|^{2} u=0 .
\end{gathered}
$$

The cross-derivative term $\partial^{2} u / \partial \zeta_{\text {loc }} \partial \tau_{\text {loc }}$ is particularly troublesome, defying straightforward interpretation and being awkward computationally. One could perhaps restrict attention to solutions with $\kappa \alpha \ll O(1)$ and $\kappa \alpha^{2} \ll$ $O(1)$, with terms at $\partial^{2} u / \partial \tau_{\text {loc }}^{2}$ and $\partial^{2} u / \partial \zeta_{\text {loc }} \partial \tau_{\text {loc }}$ assumed to be both $O(1)$. These solutions satisfy $\left[\kappa \partial^{2} /\right.$ $\left.\partial \zeta_{\mathrm{loc}}^{2}+i \partial / \partial \zeta_{\mathrm{loc}}+(s / 2) \partial^{2} / \partial \tau_{\mathrm{loc}}^{2}+|u|^{2}\right] u\left(\tau_{\mathrm{loc}}, \zeta_{\mathrm{loc}}\right)=0$, which is identical in structure to the spatial Helmholtz equation [2]. However, such a level of approximation is not in the spirit of this analysis. Since the Galilean boost serves no useful purpose, we dispense with it and deal directly with Eq. (1) instead.

The characteristics of Eq. (1), which are determined by the interplay between spatial and temporal dispersion, may be classified as relativistic [hyperbolic when $\operatorname{sgn}(s \kappa)=$ -1 ] or pseudorelativistic [elliptic when $\operatorname{sgn}(s \kappa)=+1$ ]. With these distinctions in mind, it is instructive to consider transformations in the $(\tau, \zeta)$ plane. Under the coordinate change

$$
\tau=\frac{\tau^{\prime}-V \zeta^{\prime}}{\sqrt{1+2 s \kappa V^{2}}}
$$

and

$$
\zeta=\frac{2 s \kappa V \tau^{\prime}+\zeta^{\prime}}{\sqrt{1+2 s \kappa V^{2}}}
$$

which is parameterized by velocity $V$, the covariance of Eq. (1) is guaranteed so long as $u$ transforms according to

$$
\begin{aligned}
u(\tau, \zeta)= & \exp \left[-i \frac{s V \tau^{\prime}}{\sqrt{1+2 s \kappa V^{2}}}+\frac{i}{2 \kappa}\left(1-\frac{1}{\sqrt{1+2 s \kappa V^{2}}}\right) \zeta^{\prime}\right] \\
& \times \exp \left[-i s \alpha \frac{\tau^{\prime}-V \zeta^{\prime}}{\sqrt{1+2 s \kappa V^{2}}}+i s \alpha \tau^{\prime}\right] u^{\prime}\left(\tau^{\prime}, \zeta^{\prime}\right) .(3 \mathrm{c})
\end{aligned}
$$

One then encounters the first notion of connections to special relativity - the coordinate transformation is close to the two-dimensional Lorentz form [3], but where $\tau$ plays the role of "space" and $\zeta$ is the analogue of "time." The correspondence is essentially exact when $\operatorname{sgn}(s \kappa)=-1$, in which case Eqs. (3a) and (3b) describe a skew [or a rotation when $\operatorname{sgn}(s \kappa)=+1$ ], as shown in Fig. 1 .

By considering coordinate differences $\Delta \zeta \equiv \zeta_{2}-\zeta_{1}$ and $\Delta \tau \equiv \tau_{2}-\tau_{1}$, it can be shown that $\Delta \chi^{2} \equiv \Delta \zeta^{2} /$ $2 s \kappa+\Delta \tau^{2}$ is the invariant interval between points 1 and

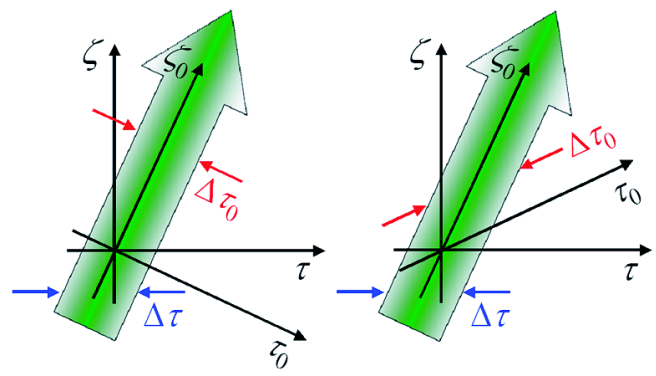

FIG. 1 (color online). Illustration of the relationship between pulse durations in the laboratory frame $(\tau, \zeta)$ and the rest frame $\left(\tau_{0}, \zeta_{0}\right)$. The two distinct spatiotemporal regimes are: (a) $\operatorname{sgn}(s \kappa)=+1$; and (b) $\operatorname{sgn}(s \kappa)=-1$. 
2 in the $(\tau, \zeta)$ plane. When $\operatorname{sgn}(s \kappa)=+1$, rotation (3) preserves a quantity $\Delta \chi^{2}$ that is essentially the geometric distance between the points (a signature of Euclidean spaces). Conversely, when $\operatorname{sgn}(s \kappa)=-1$, skew (3) does not preserve "geometric distances." In such a regime, the $(\tau, \zeta)$ plane behaves more like a Riemanian space [12].

Two successive applications of transformation (3), parameterized by $V_{0}$ and $V$, respectively, uncover the velocity combination rule for solutions of Eq. (1):

$$
W=\frac{V+V_{0}}{1-2 s \kappa V V_{0}} .
$$

This rule bears striking similarity to the addition law for particle and wave packet (group) velocities in special relativity (though one must be mindful that $V_{0}, V$, and $W$ are strictly related to inverse velocities in unscaled units). There are two other crucial points to note about Eq. (4). Firstly, it has been derived independently of any particular solution; secondly, it is independent of the Kerr effect. It thus applies to a wide range of uniform nonlinearities and, equally well, to linear systems [13].

Having considered the space-time geometry of Eq. (1), we turn attention to exact analytical solutions. For a lucid description of the consequences of spatiotemporal dispersion, we will consider only unidirectional solutions. The simplest solutions are continuous wave (cw) fields $u(\tau, \zeta)=\rho_{0}^{1 / 2} \exp [i(-\Omega \tau+K \zeta)] \exp (-i \zeta / 2 \kappa)$. Here, $\rho_{0}$ is light intensity, $\Omega$ measures the deviation of the envelope from the carrier frequency, and $K$ is a propagation constant. The dispersion relation $\kappa K^{2}-1 / 4 \kappa-$ $\Omega(\alpha-s \Omega / 2)-\rho_{0}=0$ prescribes families of ellipses or hyperbole in the $(\Omega, K)$ plane; its two solution branches are $K= \pm(2 \kappa)^{-1}\left[1+4 \kappa \rho_{0}+4 \kappa \Omega(\alpha-s \Omega / 2)\right]^{1 / 2}$. The quadratic character of the wave equation supports evolution in both forward $(+)$ and backward $(-)$ longitudinal senses, as model (1) recognizes the physical equivalence of the $+z$ and $-z$ directions [2].

It is helpful to assess the stability of $(\Omega=0) \mathrm{cw}$ solutions against small-amplitude modulations. Considering a Fourier mode with temporal frequency $\Omega_{p}$ and complex wave number $K_{p}$ (whose imaginary part allows growth of this perturbation), linear stability analysis [14] reveals that $K_{p}$ satisfies

$$
\begin{gathered}
\kappa^{2} K_{p}^{4}-\left(1+6 \kappa \rho_{0}-s \kappa \Omega_{p}^{2}\right) K_{p}^{2}+2 \alpha \Omega_{p} \sqrt{1+4 \kappa \rho_{0}} K_{p} \\
+\frac{1}{2} \Omega_{p}^{2}\left[\frac{1}{2} \Omega_{p}^{2}-2\left(\alpha^{2}+s \rho_{0}\right)\right]=0 .
\end{gathered}
$$

Equation (5) predicts a ( $\kappa$ independent) long-wave modulational instability for $s=+1$ but not for $s=-1$, as one might expect. Short-wave instabilities are more involved [and depend upon $\operatorname{sgn}(s \kappa)$ ], but they do not affect physically meaningful solutions [14].

Of more interest than cw solutions are solitons. These self-localizing and self-stabilizing pulses play a pivotal role in the understanding of waves in nonlinear systems.
The soliton solutions vividly demonstrate some key characteristics that arise from the linear wave operator involved. The governing equation admits two distinct families of exact analytical solitons. Bright families exist in the anomalous GVD regime $(s=+1)$ and are given by

$$
\begin{aligned}
u(\tau, \zeta)= & \rho_{0}^{1 / 2} \operatorname{sech}\left(\rho_{0}^{1 / 2} \frac{\tau \mp W \zeta}{\sqrt{1+2 \kappa W^{2}}}\right) \exp (i \Omega \tau) \\
& \times \exp \left[ \pm i \sqrt{1+4 \kappa \beta-4 \kappa \Omega\left(\alpha+\frac{\Omega}{2}\right)} \frac{\zeta}{2 \kappa}\right] \\
& \times \exp \left(-i \frac{\zeta}{2 \kappa}\right)
\end{aligned}
$$

where $\rho_{0}$ is peak intensity and $\beta \equiv \rho_{0} / 2$. The upper (lower) signs correspond to pulses travelling in the forward (backward) longitudinal direction. Dark families arise in the normal GVD regime $(s=-1)$. They comprise a (modulationally stable) cw background that supports a phasetopological "dip" $[2,6,10]$. For brevity, we present only the black solution here:

$$
\begin{aligned}
u(\tau, \zeta)= & \rho_{0}^{1 / 2} \tanh \left(\rho_{0}^{1 / 2} \frac{\tau \mp W \zeta}{\sqrt{1-2 \kappa W^{2}}}\right) \exp (-i \Omega \tau) \\
& \times \exp \left[ \pm i \sqrt{1+4 \kappa \beta+4 \kappa \Omega\left(\alpha+\frac{\Omega}{2}\right)} \frac{\zeta}{2 \kappa}\right] \\
& \times \exp \left(-i \frac{\zeta}{2 \kappa}\right),
\end{aligned}
$$

where $\beta \equiv \rho_{0}$. For solitons (6a) and (6b), the net velocity $W$ is given by

$$
W=\frac{\alpha+\Omega}{\sqrt{1+4 \kappa \beta-4 s \kappa \Omega\left(\alpha+\frac{1}{2} \Omega\right)}} .
$$

Pulses with $\Omega<0$ travel faster than those with $\Omega>0$, while the subset of solutions that evolve forward in time are those with $\Omega>-\alpha$ (which ensures that $W>0$ ).

When $\Omega=0$, so that the pulse envelope is centered on the carrier frequency, the intrinsic velocity is found to be $V_{0}=\alpha(1+4 \kappa \beta)^{-1 / 2}$. An application of combination rule (4) then yields $V=\left(W-V_{0}\right)\left(1+2 s \kappa W V_{0}\right)^{-1}$, whereupon substituting for $W$ [from Eq. (7)] and $V_{0}$, one finds that

$V(\Omega)$

$$
=\frac{(\Omega+\alpha) \sqrt{1+4 \kappa \beta-4 s \kappa \Omega\left(\alpha+\frac{1}{2} \Omega\right)}-\alpha \sqrt{1+4 \kappa \beta}}{1+4 \kappa \beta-2 s \kappa(\Omega+\alpha)^{2}} .
$$

One can always boost to the rest frame of the solitons by deploying transformation (3) with the velocity parameter selected to be $W$ [as given in Eq. (7)]. In that frame, the pulse duration is $\Delta \tau_{0} \equiv 2 / \rho_{0}^{1 / 2}$ while in the laboratory frame it is $\Delta \tau=\left(1+2 s \kappa W^{2}\right)^{1 / 2} \Delta \tau_{0}$, or equivalently, 


$$
\frac{\Delta \tau}{\Delta \tau_{0}}=\left[\frac{1+4 \kappa \beta+2 s \kappa \alpha^{2}}{1+4 \kappa \beta-4 s \kappa \Omega\left(\alpha+\frac{1}{2} \Omega\right)}\right]^{1 / 2} .
$$

Hence, measured pulse duration depends explicitly upon both system and solution parameters: $\Delta \tau_{0}$ is dilated to (contracted to) $\Delta \tau$ when $\operatorname{sgn}(s \kappa)=+1(-1)$, as in Fig. 1 .

It is essential that the predictions made by Eq. (1) converge with conventional pulse theory. That is, one must be able to recover asymptotic results pertaining to the approximate equation

$$
i\left(\frac{\partial u}{\partial \zeta}+\alpha \frac{\partial u}{\partial \tau}\right)+\frac{s}{2} \frac{\partial^{2} u}{\partial \tau^{2}}+|u|^{2} u \simeq 0 .
$$

When $\kappa V^{2} \rightarrow 0$, the coordinate change in Eqs. (3a) and (3b) becomes $\tau \simeq \tau^{\prime}-V \zeta^{\prime}$ and $\zeta \simeq \zeta^{\prime}$ while envelope $u$ transforms as $u(\tau, \zeta) \simeq \exp \left[-i s V \tau^{\prime}+i s\left(V^{2} / 2+\right.\right.$ $\left.\alpha V) \zeta^{\prime}\right] u^{\prime}\left(\tau^{\prime}, \zeta^{\prime}\right)$. Similarly, when $\kappa V V_{0} \rightarrow 0$ the velocity combination rule, Eq. (4), assumes Galilean form $W \simeq$ $V+V_{0}$. These results illustrate that convergence is a subtle notion, in that $\kappa \partial^{2} u / \partial \zeta^{2} \rightarrow 0$ involves more than just simply $\kappa \simeq 0$ [2]. In fact, the SVEA corresponds to a simultaneous multiple limit applied to Eq. (1) solutions. When $\kappa \rightarrow 0, \kappa \beta \rightarrow 0$, and $\kappa \Omega(\alpha+\Omega / 2) \rightarrow 0$, one recovers

$$
\begin{aligned}
u(\tau, \zeta) \simeq & \rho_{0}^{1 / 2} \operatorname{sech}\left[\rho_{0}^{1 / 2} \Theta(\tau, \zeta)\right] \\
& \times \exp \left[i \Omega(\tau-\alpha \zeta)+i\left(\beta-\frac{\Omega^{2}}{2}\right) \zeta\right]
\end{aligned}
$$

and

$$
\begin{aligned}
u(\tau, \zeta) \simeq & \rho_{0}^{1 / 2} \tanh \left[\rho_{0}^{1 / 2} \Theta(\tau, \zeta)\right] \\
& \times \exp \left[-i \Omega(\tau-\alpha \zeta)+i\left(\beta+\frac{\Omega^{2}}{2}\right) \zeta\right]
\end{aligned}
$$

for the forward solitons in Eqs. (6a) and (6b), where $\Theta(\tau, \zeta) \equiv \tau-W \zeta \simeq(\tau-\alpha \zeta)-\Omega \zeta$ since $W \simeq \Omega+\alpha$. The $\mathrm{cw}$ solutions, and predictions of their linear stability, also reduce to the desired results $[14,15]$. Solitons (11a) and (11b) are approximate solutions to Eq. (1) and exact solutions to Eq. (10); when expressed in local coordinates $\left(\tau_{\text {loc }}, \zeta_{\text {loc }}\right)=(\tau-\alpha \zeta, \zeta)$, they satisfy the canonical NLS equation $[5,6]$. One can thus regard $\left(\tau_{\text {loc }}, \zeta_{\text {loc }}\right)$ as defining a unique reference frame in which pulses with $\Omega=0$ are "at rest." Importantly, no such local time frame exists for the solutions of Eq. (1), because intrinsic velocity $V_{0}$ depends upon intensity $\rho_{0}$.

Extensive computations, using developments of earlier methods [16], reveal emergence of propagation-invariant pulses when using conventional solutions as initial conditions for Eq. (1) (see Fig. 2). These simulations, in combination with inverse scattering theory [17] and the Vakhitov-Kolokolov integral criterion [18], provide strong evidence that solitons (6a) and (6b) are robust entities with wide basins of attraction.
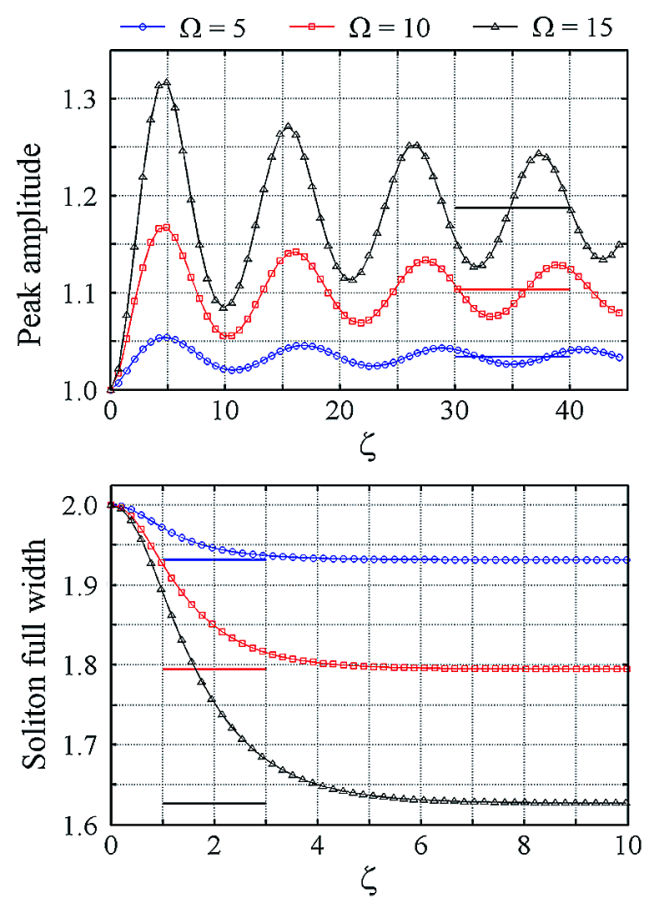

FIG. 2 (color online). Reshaping of perturbed (a) bright [with $\kappa=-10^{-3}$ ] and (b) dark with $\kappa=+10^{-3}$ ] pulses toward asymptotic stationary states (horizontal bars denote theoretical predictions) when $\alpha=1$.0. Initial conditions are exact conventional solitons with $\rho_{0}=1.0$.

In conclusion, we have analyzed a generic nonlinear wave equation describing spatiotemporal dispersion. Its mathematical structure permits one to draw parallels with special relativity (distinct from a reintroduction of the Lorentz covariance of Maxwell's equations [3]). For second-order spatiotemporal dispersion, any combination of signs and sizes of dispersion coefficients leads to either relativistic or pseudorelativistic characteristics of all system solutions (and those of the corresponding linear problem). It is intriguing to note that conventional pulse theory can be interpreted as the "low speed limit" of the more general model (similar to Newton's laws of motion emerging from relativistic mechanics).

Corrections to several conventional predictions of wave characteristics (demonstrated by $\kappa \alpha, \kappa \alpha^{2}$ and $\kappa W^{2}$ terms in soliton solutions) may be experimentally observable. Parameters $\kappa$ and $\alpha$ are independent and, in distinct physical contexts, vary over orders of magnitude. For the optical context considered [10], $\alpha$ can easily be $O\left(|\kappa|^{-1}\right)$. In optics, higher-order temporal dispersion effects can also arise. Here, we accounted only for leading terms of distinct type. This permitted exploration of distinct interplays, and a focus on general contexts and universal wave equations. We thus believe our results will have application in the description of spatiotemporal wave phenomena in other linear and nonlinear contexts. 
[1] Y. S. Kivshar and B. A. Malomed, Rev. Mod. Phys. 61, 763 (1989).

[2] P. Chamorro-Posada and G.S. McDonald, Opt. Lett. 28, 825 (2003); P. Chamorro-Posada, G. S. McDonald, and G. H. C. New, J. Mod. Opt. 45, 1111 (1998).

[3] J.D. Jackson, Classical Electrodynamics (John Wiley, New York, 1999), 3rd ed.

[4] L.F. Mollenauer, R.H. Stolen, and J.P. Gordon, Phys. Rev. Lett. 45, 1095 (1980); L. F. Mollenauer et al., Opt. Lett. 15, 1203 (1990).

[5] A. Hasegawa and F. Tappert, Appl. Phys. Lett. 23, 142 (1973); 23, 171 (1973).

[6] V.E. Zakharov and A. B. Shabat, Sov. Phys. JETP 34, 62 (1972); 37, 823 (1973).

[7] J. P. Gordon, Opt. Lett. 8, 596 (1983).

[8] Y. Kodama and A. Hasegawa, IEEE J. Quantum Electron. 23, 510 (1987); Kh. I. Pushkarov, D. I. Pushkarov, and I. V. Tomov, Opt. Quantum Electon. 11, 471 (1979).

[9] K. J. Blow and N. J. Doran, in Nonlinear Waves in Solid State Physics, edited by A. D. Boardman (Plenum Press, New York, 1990), p. 325.
[10] F. Biancalana and C. Creatore, Opt. Express 16, 14882 (2008).

[11] R. W. Boyd, Nonlinear Optics (Academic Press, San Diego, 2003), 2nd ed.

[12] H. Goldstein, Classical Mechanics (Addison-Wesley, Philippines, 1980), 2nd ed.

[13] H. Hernandez-Figueroa et al., Localized Waves (John Wiley and Sons, New York, 2008), 2nd ed.

[14] J. M. Christian, G. S. McDonald, and P. Chamorro-Posada, J. Phys. A 40, 1545 (2007).

[15] Y. S. Kivshar, D. Anderson, and M. Lisak, Phys. Scr. 47, 679 (1993).

[16] P. Chamorro-Posada, G. S. McDonald, and G. H. C. New, Opt. Commun. 192, 1 (2001).

[17] J. Satsuma and N. Yajima, Prog. Theor. Phys. Suppl. 55, 284 (1974).

[18] M. G. Vakhitov and A. A. Kolokolov, Radiophys. Quantum Electron. 16, 783 (1973); J. M. Christian et al., Phys. Rev. A 76, 033834 (2007). 